\title{
Unilateral Right Exophthalmia Revealing Systemic Sarcoidosis: A Case Report and a Review of the Literature
}

\begin{abstract}
Authors:
*Sofia Baina, Leila Achachi, Mariam El Yahiyaoui, Mustapha El Ftouh, Laila Herrak

Pulmonology department, Faculty of Medicine and Pharmacy, Med V University, Rabat, Morocco

*Correspondence to sofiabaina@hotmail.com

Disclosure: $\quad$ The authors have declared no conflicts of interest.

Acknowledgements: Dr Baina was responsible for patient management and drafted the manuscript. Prof Achachi and Prof Herrak carried out bronchoscopy and participated in patient followup. Dr Baina, Prof El Ftouh, and Dr El Yahiyaoui carried out pathology tests and were responsible for all interventional pulmonology procedures. Prof El Herrak critically revised the manuscript and gave final approval of the version. All authors read and approved the final manuscript. Written consent was obtained from the patient for publication of study.
\end{abstract}

Received: $\quad 25.04 .19$

Accepted: $\quad 05.07 .19$

Keywords: $\quad$ Corticotherapy, exophthalmia, sarcoidosis.

Citation: $\quad$ EMJ Respir. 2019 [Epub ahead of print]. DOI/10.33590/emjresp/19-00088

\begin{abstract}
Background: Sarcoidosis is only revealed in 3\% of the cases among Caucasians by ophthalmic damage and, when it does, it presupposes that the visceral impairment has remained silent so far. In this article, the exceptional case of a patient with systemic sarcoidosis revealed by unilateral exophthalmia is reported.

Case presentation: The patient is a female with no history of substantial pathology. She had a unilateral right exophthalmia and ptosis evolving over 3 years. A dyspnea and dry cough were also reported with a duration of 1 year. The chest X-ray and CT scan revealed bilateral hilar opacities and mediastinal lymphadenopathy that lead to the suspicion of sarcoidosis. The cerebro-orbital CT scan led to the classification of the patient's exophthalmia as Grade I and eliminated the possibility of other aetiologies. The mediastinoscopy indicated a granulomatous adenitis with no caseous necrosis, which allowed the diagnosis of a mediastinopulmonary sarcoidosis.
\end{abstract}

Discussion and conclusion: The diagnostic approach to exophthalmia should involve a systematic search for sarcoidosis, although this aetiology remains exceptional. 


\section{INTRODUCTION}

Sarcoidosis is a granulomatous systemic condition that can affect any organ with a predilection for the lungs and lymphatic system. Ophthalmic disorders, mostly bilateral, are reported in $25-50 \%$ of the patients with sarcoidosis and may at times reveal this systemic disease. In exceptional cases, mainly anterior uveitis, and ocular sarcoidosis in other instances, can take the form of exophthalmia. In the following case, an unusual unilateral exophthalmia revealing systemic sarcoidosis in a female patient is reported.

\section{CASE PRESENTATION}

A 65-year-old Caucasian female patient with no history of substantial pathology presented to the authors with a unilateral right exophthalmia and ptosis evolving for 3 years in an afebrile context and conservation of her general condition. The onset of exertional dyspnea and dry cough was reported for 1 year. The pleuropulmonary physical examination was without abnormalities, and the rest of the examination is not specific except for this right exophthalmia (Figure 1).

While chest X-ray showed bilateral hilar opacities, the chest CT scan revealed numerous calcified mediastinal lymphadenopathy in various locations: pre-tracheal (and bilateral), retro and precranial, left para-aortic, and intrabronchial (Figure 2).

In the framework of an aetiological evaluation, fiberoptic bronchoscopy was performed and revealed a diffuse and bilateral inflammatory aspect. Tiered bronchial biopsies and bronchoalveolar lavage (BAL) were inconclusive. BAL revealed a cell formula which comprised $79 \%$ macrophages, $17 \%$ lymphocytes, $4 \%$ neutrophils, no eosinophils, and no mast-cells. It showed that Golde score was O. No pathogens, ferruginous bodies, or tumour cells could cytologically be identified; however, minor salivary gland biopsy showed a nonspecific chronic sialadenitis.

Biologically, the immunological assessment was negative and the phosphocalcic assay had no particularities. Angiotensin-converting enzyme activity was positive and IL-2 receptor was not investigated. The phthisiological analysis was negative. The plethysmography showed a total lung capacity of $79 \%$ and the diffusion capacity of the lung for carbon monoxide was $75 \%$.

The conducted mediastinoscopy has exposed a granulomatous adenitis with no caseous necrosis (Figure 3). The ophthalmic examination was without abnormalities. Thus, the cerebro-orbital CT scan led to the classification of the patient's exophthalmia as Grade I and a calcified aspect of the left eyeball's wall.

Based on this data, the diagnosis of mediastinopulmonary sarcoidosis with exophthalmia was strongly referred and the subject was placed under systemic corticotherapy, initially at the dose of $1 \mathrm{mg} / \mathrm{Kg} /$ day then at progressively reduced doses. After approximately 2 months of treatment, substantial clinical benefits were seen through a reduction in the coughing and a slight progressive decrease of the exophthalmia. The latter could have been measured by orbital-cerebral CT scan, but there was a concern for the consequences of successive and closely spaced irradiation; this was eventually reconsidered afterwards.

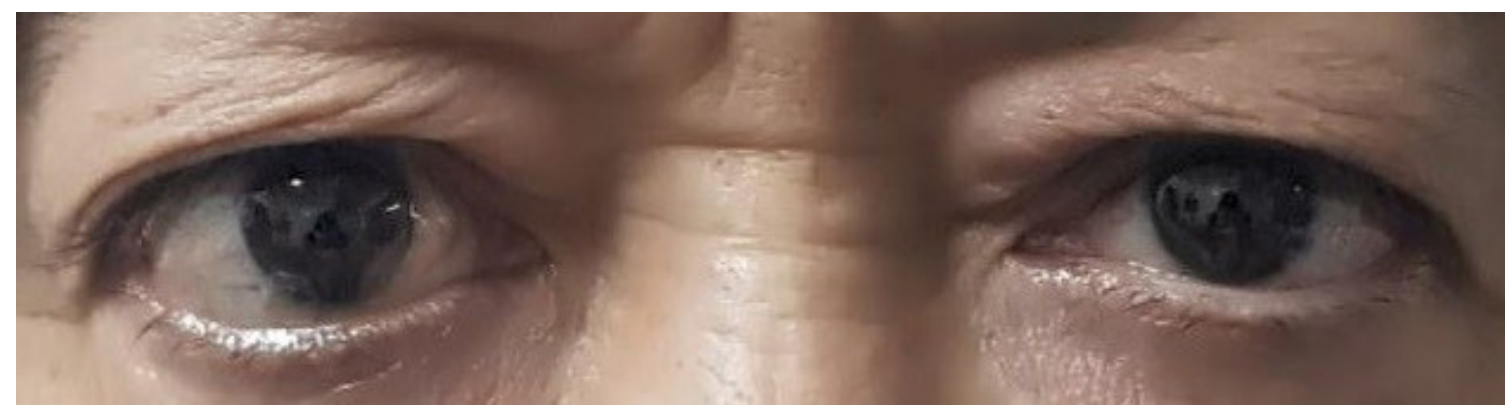

Figure 1: Patient with right exophthalmia. 

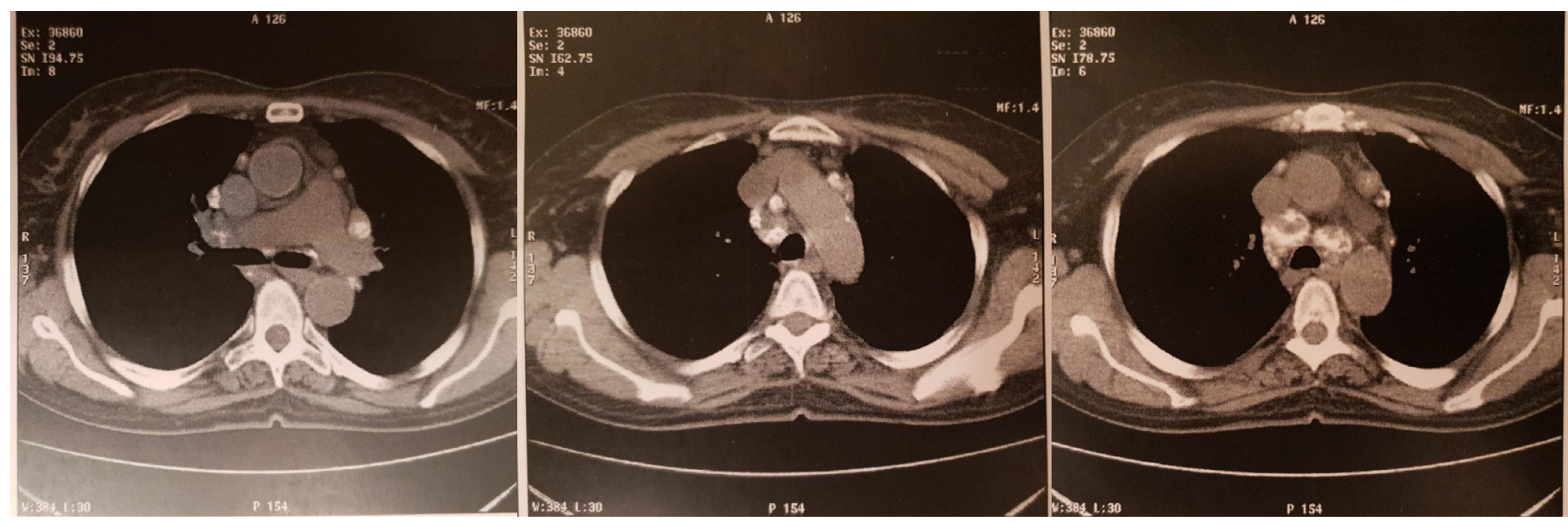

Figure 2: Chest CT scan revealing numerous calcified mediastinal adenopathy.

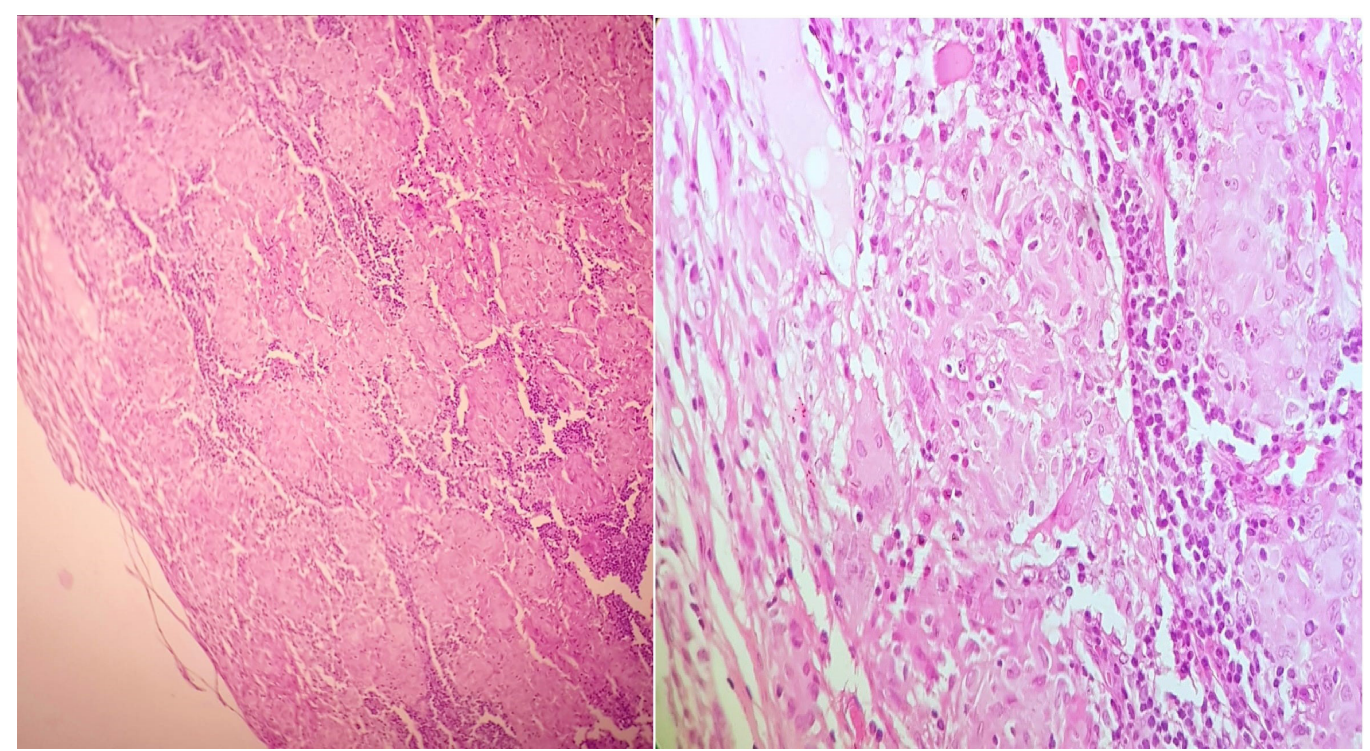

Figure 3: Medium and high magnification, haematoxylin, and eosin staining.

\section{DISCUSSION}

Sarcoidosis, also known as Besnier-BoeckSchaumann disease, is a multisystemic granulomatosis of unknown aetiology. In the study by Bezo et al., ${ }^{4}$ the average age of consultation was 48 years old (from 36-87); for others, it has been documented as 42 or 43 years old, the patient in this present study was 65 years old. Currently, there is no accurate test for sarcoidosis. Diagnosis is based upon a conjunction of three stringent criteria as defined by the American Thoracic Society (ATS), European Respiratory Society (ERS), and the World Association of Sarcoidosis and other Granulomatous Disorders (WASOG) Consensus Conference:

> A consistent medical and radiological record.

$>$ Demonstration of noncaseating granuloma.

$>$ Exclusion of other causes of granulomatosis.

In the literature, the median free interval between diagnosis and the first ophthalmic symptoms is 8 years (3-14 years). ${ }^{4}$ In this present case, the symptoms started 3 years before the disease had been diagnosed. Ophthalmic manifestations may be revealing of systemic sarcoidosis, as seen in $80 \%$ of the cases in the study of Beso et al., ${ }^{4}$ along 
with the case of the present authors' patient, who presented exophthalmia before the respiratory symptoms, leading to the accurate diagnosis.

Regarding the clinical presentation, Benouhoud et al. ${ }^{5}$ reported a case of pulmonary mediastinal sarcoidosis disclosed by a unilateral right exophthalmia, further complicated by sight loss and combined with dyspnea. Another study reported two cases: a bilateral exophthalmia combined with a bilateral maxillary sinusitis and dry cough; the second was afflicted by exertional dyspnea in conjunction with his right exophthalmia resulting in a complication of blindness. ${ }^{6}$ This study is staging a right unilateral exophthalmia with exertional dyspnea and dry cough.

Eye diseases at the origin of exophthalmia are exceptional, whether accompanied or not by systemic damage. They have only been reported a few times in literature, and granulomatous orbital infiltration leading into exophthalmia and dysmotility disorders has exceptionally been encountered. Obenauf et al. ${ }^{8}$ conducted a study involving 532 patients with sarcoidosis; only $2 \%$ presented with trouble of ocular dysmotility. This was not observed in the present authors' female patient either, which aligned with the findings of other studies. ${ }^{5,6}$ If Benouhoud ${ }^{5}$ could identify granulomatous inflammation in his patient, this was not observed in the case reported by Sabir, ${ }^{6}$ nor in this present study. Obanauf only identified it in $1 \%$ of the subjects.

The treatment of ocular sarcoidosis is mostly based on local or systemic corticotherapy at a dose of $1 \mathrm{mg} / \mathrm{kg} /$ day for 1 years, but may be needed for up to 2 years. The treatment is so effective that exeresis or reduction surgery is rarely needed. Immunosuppressants are only used as a second-line therapy when steroidsparing treatment is required in the event of corticodependency or corticoresistance. At all times, it would be necessary to prevent the potential side effects of corticoids.

The patient in this current report benefitted from systemic corticosteroids at doses of $1 \mathrm{mg} / \mathrm{kg} / \mathrm{day}$ and thereafter at progressively reduced doses. Her clinical course was positive with a slight decrease of the exophthalmia. This therapeutic approach and the clinical evolution are thus in line with the ones reported in the literature. ${ }^{5,6}$,

During the active phase of the disease, close supervision is required, and subsequently adapted to the disease progression. Multidisciplinary surveillance remains the best policy, given that the risk of disability in the ocular affection of sarcoidosis imposes optimum care.

\section{CONCLUSION}

Sarcoidosis is a multisystemic granulomatosis of unknown aetiology, of which the diagnosis is difficult to establish because it is based on a series of clinical, paraclinical, and histological arguments. The presence of epithelioid granulomas is very suggestive, although not pathognomonic. The diagnostic approach to exophthalmia should involve a systematic search for sarcoidosis, taking into consideration the therapeutic implications that arise from it, although this aetiology remains exceptional.

\section{References}

1. Valeyre D et al. Sarcoidosis. Lancet. 2014;383(9923):1155-67.

2. Baughman RP et al. Clinical characteristics of patients in a case control study of sarcoidosis. Am J Respir Crit Care Med. 2001;164(10Pt 1):1885-9.

3. Baughman RP et al. Ocular sarcoidosis. Semin Respir Crit Care Med. 2010;31(4):452-62.

4. Bezo C et al. [Ocular and neuroophthalmological sarcoidosis: A retrospective study of 30 cases]. Journal Français d'Ophtalmologie. 2013;36(6):473-80 (In French)..
5. Benouhoud $\mathrm{N}$ et al. [Unilateral exophthalmitis revealing systemic sarcoidosis]. Revue des Maladies Respiratoires. 2006;23(4):141. (In French).

6. Sabir L et al. [Systemic sarcoidosis revealed by exophthalmitis]. Revue des Maladies Respiratoires. 2007;24(HS1):139. (In French).

7. [No authors listed]. Statement on sarcoidosis. Joint Statement of the American Thoracic Society (ATS), the European Respiratory Society (ERS) and the World Association of Sarcoidosis and Other Granulomatous Disorders (WASOG) adopted by the
ATS Board of Directors and by the ERS Executive Committee, February 1999. Am J Respir Crit Care Med. 1999;160(2):736-55.

8. Papadia M et al. Diagnosis of ocular sarcoidosis. Ocul Immunol Inflamm. 2010;18(6):432-41.

9. Obenauf CD et al. Sarcoi-dosis and its ophthalmic manifestations. Am J Ophthalmol. 1978;86(5):648-55.

10. Newman LS et al. Sarcoidosis. N Engl J Med. 1997;336(17):1224-34.

11. Laghmari IM et al. [Uvaite during sarcoidosis at the child: Diagnostic difficulties about a case]. Bull Soc 
Belge Ophtalmol. 2008;307:47-51. (In French).

12. Prabhakaran VC et al. Orbital and anexal sarcoidosis. Arch Ophthalmol. 2007;125(12):1657-62.
13. Laaguili J et al. [Atypical presentation of orbital sarcoidosis]. Journal Français d'Ophtalmologie. 2015;38(9):e203-5. (In French).

14. Muller-Quernheim J. Sarcoidosis: Immunopathogenetic concepts and their clinical application. Eur Respir J. 1998;12(3):716-38.

15. Smith $\mathrm{G}$ et al. Advances in the genetics of sarcoidosis. Clin Genet. 2008;73(5):401-12. 